\title{
BENIGN BREAST DISEASE: THE CONCEPT OF ANDI
}

\author{
Wyn Morgan, Consultant Surgeon \\ Lancaster \& Kendal Hospitals
}

Ninety percent of patients with breast disorders presenting to a surgical clinic have benign breast disease and the majority of these are concerned that they may have malignant disease and wish to have that excluded. The most common forms of presentation are a mass, pain, nipple discharge or some deformity of the breast.

There has been much confusion in the terminology and nomenclature of benign breast disorders. Clinicians and pathologists may use the same terms, but the clinician often has a clinical picture in mind where the pathologist is referring to a particular histological pattern. The use of a clinical name to describe a benign condition may not correlate with the histological appearance if a biopsy is performed. For example, a clinician may diagnose fibrocystic disease of the breast, but should a biopsy of that area be carried out, there is often no histological evidence of what a pathologist would call fibrocystic disease. In addition, that biopsy may show one histological pattern in one area and a different histological pattern in an adjacent area. Furthermore, a histological examination of a section of breast from a woman who is asymptomatic and who has no overt breast disease may show a spectrum of histological appearances. The 'fibrosis' and 'adenosis' that may be seen on a biopsy from a symptomatic portion of breast may also be seen in a biopsy of the same breast where there is no clinical complaint.

Over the years there have been many different names for common benign breast disorders. The familiar cyclical nodularity that we see in the breast has been called fibrocystic disease, fibroadenosis, cystic hyperplasia, hyperplastic cystic disease, Schimmelbusch's disease, chronic cystic mastitis and cystic mastopathy. In addition, duct ectasia/periductal mastitis has been termed plasma cell mastitis, varicocele tumour, comedo mastitis, mastitis obliterans and secretory disease. Giant fibroadenomatous tumours of the breast have been called giant fibroadenoma, cystosarcoma phylloides, phylloides tumour, juvenile fibroadenoma and sero-cystic disease of Brodie.

The breast is under endocrine control and therefore shows a wide range of appearances associated with cyclical levels of hormone secretion. In addition, the cyclical changes are set against a background of development and involution of the breast which occur at the extremes of reproductive life. Benign breast disorders arise when the changes extend outside the range of normal. At times, this can be difficult to define. The epithelial and stromal elements of the breast lobule are under hormonal control and normality depends on a balanced relationship between these two elements. Interference with this relationship may be responsible for many of the conditions which are termed benign breast disease. Superimposed on these cyclical changes are the radical effects of pregnancy and lactation. The repeated development and involutional changes of menstruation and pregnancy occuring over 40 years of reproductive life lead to minor aberrations in the structure of the breast lobules and stroma. In pregnancy, for example, the intense hormonal stimulation leads to lobular development, but involution occurs in a patchy fashion after lactation is finished.

The majority of benign disorders of the breast can be explained by the concept of $\mathrm{ANDI}^{(1)}$, which stands for 'Aberrations of Normal Development and Involution'. ANDI also provides an age-related diagnostic framework which is useful for the clinician in the diagnosis of breast disorders. Particular conditions of the breast correlate closely with the patient's age, and the concept of ANDI helps the clinician to understand the changes that are likely to be occuring in the patient's breasts at that stage of her life. If the ANDI concept is applied to duct ectasia/periductal mastitis, epithelial hyperplasia and other conditions with a well-defined aetiology, a framework for the whole spectrum of benign breast disorders is provided.

\section{LESIONS ASSOCIATED WITH BREAST DEVELOPMENT}

1 Fibroadenoma The most common lesion seen in relation to breast development is the fibroadenoma. This is an aberration of normal lobular development rather than a tumour. These lesions develop from the breast lobule and the lobular origin explains many features of fibroadenoma - eg it is most common in young women at the time of maximal lobular development and the intralobular stroma form a major element in fibroadenomas. Clinically the lesion is predominantly a tumour of young women, but studies have shown that the median age of diagnosis is approximately 30 years. Fibroadenomas probably become clinically apparent in the third or fourth decade, either because the fibroadenoma enlarges or because the breast itself becomes softer or more pendulous. In the older age groups, the typical signs of a fibroadenoma may be obscured by involutional change. A few fibroadenomas show rapid growth reaching a large size. They have the growth and histological features of a simple fibroadenoma and behave in a benign fashion. These giant fibradenomas have a bimodal distribution and are seen at the extremes of reproductive life, ie in the 13 - to 18 -year-old age group and around the time of the menopause. After the menopause, fibroadenomas are uncommon which suggests that they involute with the remainder of the breast tissue. In this process they often calcify.

The aetiology of fibroadenoma is unknown, but the fact 
that lobular proliferation is a response to oestrogen stimulation suggests that they occur as a result of a lobule becoming abnormally sensitive to oestrogen. Fibroadenomas grow by proliferation of the lobule and also involvement of the adjacent lobules. This fact may explain the significant recurrence rate after removal. Fibroadenomas are often multiple in the breast and there is some evidence that their incidence is increasing in western populations. There is conflicting evidence whether the use of the contraceptive pill increases or decreases the incidence of fibroadenomas. They are most common in the left breast and especially in the upper outer quadrant. The fibroadenoma will respond to external influences as will normal breast tissues. They will undergo hyperplasia during pregnancy, secrete milk during lactation and involute in the menopause.

In a young woman the typical fibroadenoma is a smooth, round or lobulated discrete lump which is very mobile. Occasionally, fibroadenomas arise behind the nipple and their mobility is limited by the surrounding duct tissue. The marked mobility of a fibroadenoma is due to encapsulation which is well defined. In the elderly population, a fibroadenoma is occasionally discovered as a small, hard discrete mass which is often calcified.

2 Adolescent hypertrophy Another lesion associated with breast development is adolescent hypertrophy of the breast. This is due to excessive stromal hyperplasia. The aetiology is not known.

\section{UNDERSTANDING CYCLICAL CHANGE}

\section{Mastalgia and nodularity}

Normal cyclical change in the breast involves premenstrual enlargement and tenderness and a postmenstrual resolution of theses symptoms. Some tender nodularity of the breast is therefore part of the normal menstrual cycle. This becomes clinically abnormal when there is sufficient cyclical discomfort to become pain and this is referred to as mastalgia. This is accompanied by cyclical nodularity. There are no histological changes that have been clearly defined for this condition. Cyclical pronounced mastalgia or severe painful nodularity are terms that have been used to differentiate this disorder from normal physiological cyclical discomfort and lumpiness. In the past this condition has often been called fibrocystic disease or fibroadenosis. However, the clinical syndrome is not necessarily associated with either the presence of fibrosis or cysts within the breast. There is some evidence from hormonal studies that there is an underlying physiological abnormality related to excess prolactin release from the pituitary following the stimulation of the hypothalmic-pituitary axis. Cyclical painful nodularity of the breast occurs predominantly during the reproductive years (age 20 to 35 ) and gradually merges into those symptoms related to involution of the breast.

\section{INVOLUTIONAL CHANGE}

\section{Cysts}

The characteristic disorder of breast involution is cyst formation. Involution of the breast is a fluctuating process which extends over a period of twenty years. There is evidence that the normal epithelial involution of the breast lobule is dependant on the maintained presence of the specialised stroma surrounding the lobule. When the stoma involutes rapidly leaving the epithelial acini then microcyst formation occurs. Macrocysts form on the basis of the development of microcysts probably due to the obstruction of the efferent ductule. The idea that breast cysts arise as a consequence of involution correlates with the known facts that breast cysts are common, frequently multiple and often subclinical as is more commonly recognised by the increased use of mammography.

\section{Sclerosing adenosis}

Sclerosing adenosis produces a complex histological picture which shows proliferative and involutional changes. Using the concept of ANDI, sclerosing adenosis can be regarded as an abberation of either the cyclical or involutional phase of breast activity. The epithelial acini are strangled and distorted by the presence of the excess fibrous stroma.

The changes associated with duct ectasia and peri-ductal mastitis form the second major group of breast disorders. Haagensen $^{(2)}$ proposed that dilatation of the ducts was the primary event leading to stagnant secretions in the breast ducts, followed by ulceration of the duct epithelium and

\begin{tabular}{|c|c|c|}
\hline $\begin{array}{l}\text { Period of reproductive life } \\
\text { Development }\end{array}$ & $\begin{array}{l}\text { Benign breast disorder } \\
\text { NIpple inversion } \\
\text { Single duct obstruction } \\
\text { Fibroadenoma } \\
\text { Adolescent hypertophy }\end{array}$ & $\begin{array}{l}\text { Benign breast disease } \\
\text { Mammary duct } \\
\text { Fistula } \\
\text { Giant form } \\
\text { Severe form }\end{array}$ \\
\hline Cyclical change & $\begin{array}{l}\text { Mastalgia } \\
\text { Nodularity } \\
\text { Focal } \\
\text { Diffuse } \\
\text { Benign papilloma }\end{array}$ & Severe form \\
\hline Pregnancy and lactation & $\begin{array}{l}\text { Blood-stained nipple discharge } \\
\text { Galactocele and inappropriate lactation }\end{array}$ & \\
\hline Involution & $\begin{array}{l}\text { Cysts and sclerosing adenosis } \\
\text { Nipple retraction } \\
\text { Duct ectasia } \\
\text { Simple hyperplasias }\end{array}$ & $\begin{array}{l}\text { Periductal mastitis/suppuration } \\
\text { Lobular/ductal hyperplasias } \\
\text { with atypia } \\
\text { Intracystic papilloma }\end{array}$ \\
\hline
\end{tabular}


leakage of duct secretions into the peri-ductal tissue giving rise to a chemical inflammatory process. The inflammation in turn leads to peri-ductal fibrosis with consequent fibrous contraction and nipple retraction. However, Hughes et al $l^{(1)}$ consider this sequence of events to be incorrect. In particular, the sequence does not correlate with the age distribution of changes seen clinically. Duct ectasia with nipple discharge is most common in the peri-menopausal age group. The inflammatory changes and associated complications usually occur in an earlier age group including the 20's and 30's. They postulate that the primary process is a peri-ductal mastitis, possibly autoimmune in origin, leading to the destruction or weakening of the myo-epithelial layer of the ducts followed by secondary dilation of those ducts. Periductal fibrosis can occur in the absence of duct ectasia or duct inflammation and may be part of the normal involutional process in the breast. There is also some evidence that bacteria in the breast ducts may play a role in peri-ductal mastisis. However, this may be secondary rather than primary. The clinical features of nipple discharge, nipple retraction, inflammatory masses and abscesses (sterile or bacterial) are associated with duct ectasia and peri-ductal mastitis.

Simple benign epithelial hyperplasias can be considered benign breast disorders. Lobular and intraductal papillary hyperplasias are common in the pre-menopausal period and regress spontaneously after the menopause. Autopsy studies have shown that $59 \%$ of women over the age of 70 have some degree of epithelial hyperplasia, without any associated evidence of in situ carcinoma or invasive carcinoma. However, atypical lobular/ductal hyperplasias, especially those seen in the terminal duct lobular unit can be associated with malignant change and may be a pre-malignant condition.

Other common benign disorders of the breast can also be considered using the concept of ANDI (see Table):

(a) Abnormal development of the terminal breast ducts may result in nipple inversion

(b) Obstruction may lead to recurrent subareolar abscesses and the formation of a mammary duct fistula which is the common form of periductal mastitis seen in younger women

(c) Hyperplasia of the breast duct epithelium occurs in pregnancy and the papillary projections can give rise to bilateral bloody nipple discharge. This is a benign condition when occurring in pregnancy

(d) Benign duct papilloma is another condition which can result in a blood stained nipple discharge. This condition arises during the period of cyclical activity and is not thought to have any potential for malignant change. This can be regarded as an aberration of cyclical activity.

\section{Management of benign breast disease}

It is not possible to cover adequately the management of benign breast disease in a single article. Each condition would require a full chapter by itself. However, fibroadenomas and breast cysts are common, and a brief scheme for their management follows:

Under the age of 35 , a solitary discrete lump which is a fibroadenoma on clinical examination should be examined by cytology and ultrasound ${ }^{(3)}$. Senstitivites of $84 \%$ and $98 \%$ can be obtained by cytology and ultrasound respectively for the diagnosis of fibroadenoma. If all three diagnostic modalities support a diagnosis of fibroadenoma, then an expectant policy may be pursued. However, follow-up of the lesion will be necessary. Patient preference will influence this decision. If these tests do not fully support the diagnosis of a fibroadenoma, or if the lesion increases in size, then it should be excised and submitted to histology. Over the age of 35 such lesions should be excised and submitted to histology and mammography should be done. The chances of misdianosing a carcinoma as a fibroadenoma increase after the age of 35 .

Breast cysts are the most common in perimenopausal women. All breast cysts in menopausal women, especially those not taking hormone replacement therapy, should be regarded with suspicion. The majority of breast cysts can be managed by cyst aspiration ${ }^{(4)}$. However, if blood stained fluid is obtained, or if there is a residual mass, or if the cyst refills on more than two occasions, then the cyst should be more fully evaluated. The methods used would include cytology of the aspirated fluid, ultrasound and mammography. Excision of the lesion with its submission to full histology may be necessary. Cystic degeneration of a high grade malignancy and intra-cystic papillary carcinoma can masquerade as benign breast cysts.

\section{REFERENCES}

1 Hughes L E, Mansel R E, Webster D J T Benign disorders and diseases of the breast. Concepts and clinical management. Bailliere Tindall 1989

2 Haagensen C D Mammary duct ectasia - a disease that may simulate carcinoma. Cancer 1951; 4:749-761

3 Carty, N. J., Carter C, Rubin, C et al. Management of fibroadenoma of the breast. Ann R Coll Surg England 1995; 77: $127-130$

4 Ravichandran D, Carty N J, Al-Talib R K, et al. Cystic carcinoma of the breast: a trap for the unwary. Ann Roy Coll Surg England 1995; 77:123-126 